\title{
Functional Positioning of City-level Top Three Hospitals based on Grading Diagnosis and Treatment
}

\author{
Chunyan Dai, Mingqiong Wang
}

Qujing Medical College, Qujing, Yunnan, 655000, China

Keywords: Grading Diagnosis, Prefecture-level top three hospital, Functional Position

\begin{abstract}
Through the perspective of grading diagnosis and treatment, the status quo and hierarchical division of public hospitals were examined, the problems and shortcomings of current functional positioning were found, and the impact of medical order was analyzed. The functional evolution of public hospitals should follow the principle of hierarchical and differentiated development. Hospitals at all levels need to be relocated under the background of hierarchical diagnosis and treatment.
\end{abstract}

\section{Introduction}

The basic functions of the hospital include prevention, health care, medical care and rehabilitation. Specifically, medical institutions use medical science and technology to provide medical, preventive, health care and rehabilitation services to patients, specific populations or healthy people. The function positioning of hospitals should be oriented to the actual health service needs of the population in the region. With the principle of optimizing and efficient medical resources allocation, it can provide fair basic medical services, effectively control medical costs, and provide suitable and continuous benefits for the masses. Health protection and services. This study focuses on the grading and functional orientation of public hospitals in China. This is of great significance for constructing a rational grading diagnosis and treatment pattern and forming an orderly diagnosis and treatment order to solve the problem of "difficult to see a doctor and expensive to see a doctor". Public hospitals are the main components of China's medical and health service system, providing most of the medical services for the people.

\section{Proposal of functional positioning of public hospitals}

The functional orientation of public hospitals in China has been relatively vague, and the functions and division of labor between inter-level, comprehensive and specialist, inter-regional medical institutions are not clear, resulting in the re-distribution of medical resources and the development of dislocation between institutions, resulting in "big hospitals see small". The disease, the small hospital can see the distortion of the pattern. The research on the function of medical institutions in the existing literature mainly comes from two dimensions. The first is the perspective of functional differentiation between hospital levels. Due to the unreasonable allocation of health resources in large cities, the function of large hospitals and community health service centers is unclear. The medical services of large hospitals are too wide, and the functions of community health service centers are shrinking. Through the study of the relationship between the primary medical institutions and their functions, it is found that the functional orientation and relationship among the primary medical institutions in China is not clear, especially the functional orientation of township hospitals is the most vague. Due to the functional positioning defects of the urban community medical institutions in China and the unfavorable factors brought about by them, the selection rate of the first-time medical treatment for the community medical institutions is not high. Second, public hospitals have specialized functions, specialization, and segmentation perspectives. The main functions of public hospitals in China include six functions: medical services, social services, medical education, scientific research, command tasks and public health; through outpatient consultation and treatment of medical institutions in different economic categories and levels in 
Zhuhai City In the case investigation and research, the researchers defined that the basic hospitals can provide 83 basic medical services and 53 common basic medical treatments; the incentive mechanism based on the prepaid method is basically consistent with the functional orientation of the primary medical institutions. It can effectively promote the health management function of primary medical institutions.

Although there are a certain number of documents on the function and location of Chinese hospitals, most of them are based on the level and category of medical institutions, and the perspectives are relatively simple. Among them, there are the most research literatures on the function and orientation of primary medical institutions, and there are relatively few literatures on functional positioning of secondary and tertiary hospitals. There are few researches on the coordination and integration of functions among medical institutions, and there is a lack of functional positioning between medical institutions. Research and analysis of the relationship between the formation of medical order. The author explores the study of the function and orientation of medical institutions from the perspective of grading diagnosis and treatment, and focuses on the functional positioning of public hospitals at all levels and the impact on the order of medical treatment and the repositioning of medical institutions under the grading diagnosis and treatment pattern.

\section{Classification and functional positioning of public hospitals in China}

The characteristics of the current medical institution grading management: First, the main body of the hospital classification. The main body of the current classification is the health and family planning administrative department. As the "big dean" of health management, it is both an athlete and a referee, which leads to a strong administrative color of classification. Each hospital implements "level management" with reference to government agencies; Stereotype. The basis of classification of hospitals in China is in accordance with health administrative laws and regulations. The logical starting point is the existing medical institution development pattern and the positioning of the medical institution's own functional attributes, the lack of regional health planning functions and future sustainable development functions; the third is the mechanization of functional classification. The classification and classification of medical institutions is not based on the perspective of health needs, and lacks considerations for human care, medical treatment and order. Grassroots hospital. Grassroots hospitals in China include primary hospitals, community health service centers, and township health centers. The overall functional orientation is to maintain the basic health of local residents, use basic medicines, appropriate technologies and equipment, and carry out basic health and health services that are compatible with their functional positioning, including basic medical care, preventive health care, and public health. Secondary hospital. As a medical and health service center in the county, the county-level public hospital is positioned as the leader of the rural three-level medical and health service network and the hub of the urban and rural medical and health service system. It is an important platform and carrier for providing basic medical and health services to the residents in the county. The Opinions on Promoting Comprehensive Reform of County-Level Public Hospitals (National Guardian Reform defines its functions as follows: taking on common diseases and frequently-occurring diseases and treatments of county residents; critically ill and difficult disease referrals ; Rural grassroots medical and health institutions personnel training guidance; undertake some public health service functions, including disease prevention, health education, etc.; natural disasters and emergency public health emergency response. The function of urban secondary hospitals is to diagnose and treat common diseases and frequently-occurring chronic diseases in the region; patients with acute disease recovery during the referral of tertiary hospitals, patients with postoperative recovery period and patients with stable critical illness; undertake some public health services and family planning services Function.

Third-level hospital. In 2016, the National Health and Family Planning Commission issued a series of policy papers on promoting graded diagnosis and treatment, and positioned the main functions of urban tertiary hospitals to provide diagnosis and treatment services for critically ill and difficult diseases. The specific function of the tertiary general hospital is to provide high-level 
clinical special medical services to several areas in the region, to provide outpatient and inpatient services for critically ill and difficult diseases in the region; to provide regional emergency first aid and emergency medical rescue for major disasters; Provide two-way referral service, accept the referral needs of lower-level hospitals, transfer patients during rehabilitation period; undertake business technical guidance, personnel training, professional counterpart training, continuing medical education for lower-level hospitals; undertake standardized training of medical graduates Mission; complete the teaching and training of high-end medical professionals; undertake clinical research projects above the provincial level.

\section{Rational thinking on the functional orientation of public hospitals in China based on graded diagnosis and treatment}

Functional differentiation between different levels of hospitals is a necessary condition for orderly diagnosis and treatment, and is the only way for graded diagnosis and treatment. The functional orientation of public hospitals in China has problems such as overlapping functions, unclear powers and responsibilities, and unclear boundaries. Among the functional definitions of primary medical institutions and secondary medical institutions, there are common diseases, frequently-occurring diseases, and chronic diseases in basic medical services, but there is no clear distinction between the two; and grassroots, first, second and third-grade hospitals all mention health. The functions of education and public health have a vague definition of functions and a lack of distinction. The distinction between the functions of medical institutions is not clear, and it is easy to mislead the public. When patients choose to seek medical treatment, they often have a tendency to "higher". Especially, the channels for urban residents to reach large hospitals are convenient and fast, and often cause "overcrowding" in large hospitals. Therefore, setting up the diagnosis and treatment catalogue of common diseases and frequently-occurring diseases, and defining the concept connotation and extension of common diseases and frequently-occurring diseases in primary hospitals and secondary hospitals, and implementing the differentiation of functional orientations of public hospitals at all levels can realize Order medical treatment, graded diagnosis and treatment.

The process and standardization of disease diagnosis and treatment is an important part of continuous medical treatment. Whether it is the "health gatekeeper" in the United Kingdom or the "family doctor appointment" in the United States, patients are treated as a "full process". Patients should follow Seek medical treatment path and procedure for medical treatment, otherwise it will be subject to good faith or corresponding punishment measures. The setting of the diagnosis and treatment path should also fully consider the functional positioning among the medical institutions. Only a rational and orderly path model based on the differentiation of hospital functions can form a scientific and reasonable "medical logic", thus affecting the patient's choice of medical treatment. To guide patients to the first floor of the primary level, orderly medical treatment, to achieve the goal of "continuous medical care." Common diseases, frequently-occurring diseases, and chronic diseases occupy a large number of high-quality medical resources in tertiary hospitals. It is very valuable to design and formulate their diagnosis and treatment routes and treatment procedures. It can sink a large number of general diseases to the grassroots and strengthen primary medical care. Institutional vitality. The critically ill critical illness can be smoothly transferred to the tertiary hospital through the "screening" after the level-by-level referral, which enhances the "diagnostic value" of the tertiary hospital and avoids the waste of high-quality medical resources.

In the context of grading diagnosis and treatment, the functional orientation of medical institutions should be able to be "hierarchical, differentiated, differentiated, process-based, and interactive", and to achieve the functional value regression and value function maximization at all levels, all levels of hospitals The functional intersection can be promoted step by step, reflecting the differentiation of medical services between levels. Therefore, to achieve the maximization of their own value functions, all levels of medical institutions need to reposition the functions of public hospitals at all levels, and optimize the medical resource allocation of medical institutions at all levels according to the logic of "Tianji Racing”. Large hospitals should achieve functional value 
regression, secondary hospitals must realize value function optimization, and primary hospitals and primary medical institutions must realize value function reshaping. The tertiary public hospitals should realize the advanced functions of the "top level" of medical institutions: high-level medical treatment technology implementation functions; high-end medical research functions; advanced medical personnel training functions. The secondary public hospitals should realize the general functions of the "middle layer" of regional medical institutions: relatively complex common diseases, frequently-occurring diseases, chronic disease diagnosis and treatment services; regional medical emergency functions; excessive referral of difficult diseases; and some public health functions. Primary medical institutions should realize the functions of "healthy gatekeepers" as "primary" of medical institutions: prevention, treatment and treatment of basic diseases such as common diseases, frequently-occurring diseases and chronic diseases; treatment of patients during rehabilitation; health education and promotion; basic public health services Features, etc.

\section{Conclusion}

The functions of public health services, family planning services, health education and health promotion of primary medical institutions are accepted and recognized by patients, but the recognition and acceptance of basic medical services are not high. The over-confidence and over-recognition of general diseases in the second and third-level hospitals have caused functional cognitive biases in tertiary hospitals, which have largely affected the positioning and evolution of medical institutions. In addition, the functional orientation of the hospital affects the medical workers' own positioning, value identification, organizational attribution, organizational positioning, etc. In turn, the value orientation of medical workers will strengthen the development direction and final functional positioning of medical institutions.

\section{Acknowledgements}

Fund Project: Research Fund: project of scientific research foundation of Yunnan Provincial Department of education in 2016(2016ZZX278)

\section{References}

[1] Chen Yu, Wang Shengyong, Zeng Zhiwei, et al. Definition of basic diseases and basic diagnosis and treatment projects: Zhuhai experience [J]. China Health Policy Research, 2009 (1): 54-59.

[2] Xue Huanmei. Functional Positioning Analysis of Urban Medical Service Organizations [J]. Labor Security World, 2009(4): 81-83.

[3] Peng Yingchun, Wang Xiaoyan, Peng Yu, et al. Discussion on the Functional Orientation of Rural Primary Medical Institutions under the Background of New Medical Reform [J]. Chinese Medical Ethics, 2012(1): 57-59.

[4] Chen Qikun, Chen Yankui, Yang Yang, et al. Analysis of the defects in the functional orientation and access system of urban community medical institutions [J]. Modern Hospital Management, 2013(4): 25-26.

[5] Dai Tao, You Chuanmei. Functional Operation and Reform of Public Hospitals in China [J]. Chinese Health Policy Research, 2009(8): 14-20. 